\title{
Metric Invariance Entropy and Relatively Invariant Control Sets
}

\author{
Fritz Colonius
}

\begin{abstract}
For control systems in discrete time, this paper considers metric (i.e., measure-theoretic) invariance entropy for a subset $Q$ of the state space with respect to a conditionally invariant measure. The main result shows that this entropy is already determined by certain subsets of $Q$ which are characterized by controllability properties.
\end{abstract}

\section{INTRODUCTION}

Metric invariance entropy for subsets of the state space is a measure-theoretic analogue of the notion of invariance entropy of deterministic control systems, cf. [9] and [6], henceforth called topological invariance entropy. The present paper discusses its relations to controllability properties. We consider control systems in discrete time of the form

$$
x_{k+1}=f\left(x_{k}, u_{k}\right), k \in \mathbb{N}=\{0,1, \ldots\},
$$

where $f: M \times \Omega \rightarrow M$ is continuous and $M$ and $\Omega$ are metric spaces. For an initial value $x_{0} \in M$ at time $k=0$ and control $u=\left(u_{k}\right)_{k>0} \in \mathcal{U}:=\Omega^{\mathbb{N}}$ we denote the solutions by $\varphi\left(k, x_{0}, u\right), k \in \mathbb{N}$. The system should be kept in a given subset $Q$ of $M$. The notion of topological invariance entropy $h_{i n v}(Q)$ describes the average data rate needed to keep the system in $Q$ (forward in time). It is constructed with some analogy to topological entropy of dynamical systems. We refer, e.g., to [13] or [12] for the entropy theory of dynamical systems. A major difference of entropy in a control context to entropy for dynamical systems is that the minimal required entropy for the considered control task is of interest, instead of the total entropy generated by the dynamical system. Presumably, the earliest connection between control for deterministic systems and ergodic theory has been established in reference [5] where it is shown that quantized feedbacks for stabilization may lead to nontrivial invariant measures, hence chaotic systems. The contribution [7] considers measure-theoretic notions of entropy in the context of fundamental limitations in control. Here, however, the uncertainty arises due to disturbances, called conditional dither. The present paper considers properties of a metric invariance entropy with respect to quasi-stationary measures or, more generally, to conditionally invariant measures on $Q$. The notion of metric entropy employed here is a modification (and simplification) of the one introduced in [3]. It is shown that this entropy is bounded above by the topological invariance entropy and that it already determined by invariant $W$-control sets, where $W$ is the interior of $Q$. A general reference to quasi-stationary measures is the monograph [2]; the survey [8] presents, in particular, applications

This work was supported by DFG grant Co 124/19-1

Institut für Mathematik, Universität Augsburg, 86159 Augsburg, Germany fritz.colonius@math.uni-augsburg.de to population dynamics where quasi-stationary distributions may correspond to plateaus of mortality rates. In general, quasi-stationarity may occur, when exit from $Q$ occurs with probability one, but only after a long time, so that in the meantime a quasi-stationary behavior may develop.

Control sets (i.e., maximal subsets of approximate controllability) for systems in discrete time have, in particular, been analyzed in references [1], [11], [10].

The contents of this paper is as follows: First the definition of metric invariance entropy is reviewed and it is shown that the topological invariance entropy is an upper bound. Then conditions are given ensuring that the metric invariance entropy is already determined on invariant subsets in $Q$ and, finally, such invariant subsets are constructed using controllability properties.

\section{DEFINITION OF METRIC INVARIANCE ENTROPY}

In this section, we present the definition of metric invariance entropy, which has some similarity to metric entropy for dynamical systems.

Let $\mu$ be a probability measure on a space $X$ endowed with a $\sigma$-algebra $\mathfrak{F}$. For every finite measurable partition $\mathcal{P}=\left\{P_{1}, \ldots, P_{q}\right\}$ of $X$ into measurable sets the entropy is defined as

$$
H_{\mu}(\mathcal{P})=-\sum \mu\left(P_{i}\right) \log \mu\left(P_{i}\right) .
$$

The entropy specifies the expected information gained from the outcomes in $\mathcal{P}$ of an experiment or the amount of uncertainty removed, upon learning the $\mathcal{P}$-address of a randomly chosen point. Now let us look at a dynamical system (in discrete time) generated by a continuous map $T$ and its iterations on a compact metric space $X$. Here a natural choice of the relevant $\sigma$-algebra is the Borel $\sigma$-algebra $\mathcal{B}(X)$, i.e., the smallest $\sigma$-algebra containing all open subsets of $X$. For two collections $\mathfrak{A}$ and $\mathfrak{B}$ of sets let the join be defined by

$$
\mathfrak{A} \vee \mathfrak{B}:=\{A \cap B \mid A \in \mathfrak{A} \text { and } B \in \mathfrak{B}\},
$$

and for a finite measurable partition $\mathcal{P}$ of $X$ and $j \in \mathbb{N}$ let

$$
T^{-j} \mathcal{P}:=\left\{T^{-j} P \mid P \in \mathcal{P}\right\} .
$$

Then one finds that

$$
\mathcal{P}_{n}:=\bigvee_{j=0}^{n-1} T^{-j} \mathcal{P}=\mathcal{P} \vee T^{-1} \mathcal{P} \vee \ldots \vee T^{-(n-1)} \mathcal{P}
$$

again is a finite measurable decomposition of $X$. Here a natural choice for the probability measure $\mu$ is an invariant 
measure, that is,

$$
\mu\left(T^{-1} E\right)=\mu(E) \text { for all measurable } E \subset X .
$$

Then $\frac{1}{n} H_{\mu}\left(\mathcal{P}_{n}\right)$ is the per-iterate gain of information of the dynamical system $T$ with respect to $\mathcal{P}$. The entropy of $T$ with respect to the partition $\mathcal{P}$ is

$$
h_{\mu}(T, \mathcal{P}):=\lim _{n \rightarrow \infty} \frac{1}{n} H_{\mu}\left(\mathcal{P}_{n}\right),
$$

and the metric entropy of $T$ is

$$
h_{\mu}(T):=\sup _{\mathcal{P}} h_{\mu}(T, \mathcal{P}),
$$

where the supremum is taken over all finite measurable partitions $\mathcal{P}$ of $X$.

This concept has to be modified for invariance of sets in control. With the shift $\theta: \mathcal{U} \rightarrow \mathcal{U},\left(u_{k}\right) \mapsto\left(u_{k+1}\right)$, control system (1) can equivalently be described by the skew product map

$$
S: \mathcal{U} \times M \rightarrow \mathcal{U} \times M,(u, x) \mapsto\left(\theta u, f\left(x, u_{0}\right)\right) .
$$

Then $S^{n}(u, x)=\left(\theta^{n} u, \varphi(n, x, u)\right), n \in \mathbb{N}$. We suppose that a closed set $Q \subset M$ is given. A conditionally invariant measure $\mu$ for the map $S$ with respect to $Q$ is a probability measure on the Borel $\sigma$-algebra of $\mathcal{U} \times M$ such that $0<$ $\rho:=\mu\left(S^{-1}(\mathcal{U} \times Q) \cap(\mathcal{U} \times Q)\right) \leq 1$ and

$$
\rho \mu(B)=\mu\left(S^{-1} B \cap(\mathcal{U} \times Q)\right) \text { for } B \in \mathcal{B}(\mathcal{U} \times M) .
$$

Interesting conditionally invariant measures can be constructed in the following way. Fix a probability measure $\nu$ on the Borel $\sigma$-algebra $\mathcal{B}(\Omega)$ of the control range $\Omega$. Let $p(x, A)=\nu\{\omega \in \Omega \mid f(x, \omega) \in A\}, x \in M, A \subset M$, be the associated Markov transition probabilities. A quasistationary measure with respect to a closed subset $Q$ of $M$ is a probability measure $\eta$ on $\mathcal{B}(M)$ such that $0<\rho:=$ $\int_{Q} p(x, Q) d \eta \leq 1$ and

$$
\rho \eta(A)=\int_{Q} p(x, A) d \eta \text { for all } A \in \mathcal{B}(Q) .
$$

The measure $\eta$ is stationary, if and only if $\int_{Q} p(x, Q) d \eta=$ 1. This defines a probability measure on the product space $\mathcal{U} \times M$ : With the product measure $\nu^{\mathbb{N}}$ on $\mathcal{U}=\Omega^{\mathbb{N}}$ the measure $\mu=\nu^{\mathbb{N}} \times \eta$ is a probability measure satisfying (4) with $\rho=\int_{Q} p(x, Q) d \eta$, cf. [3, Proposition 2.8]. Results on the existence of quasi-stationary measures are given, e.g., in [2, Proposition 2.10 and Theorem 2.11] and [3, Theorem 2.9].

Throughout we fix a conditionally invariant measure $\mu$ with constant $\rho=\rho(\mu)$ for $Q \subset M$. We write $S_{Q}:=$ $S_{\mid \mathcal{U} \times Q}: \mathcal{U} \times Q \rightarrow \mathcal{U} \times M$ for the restriction. Then the condition in (4) can be written as $\rho \mu(B)=\mu\left(S_{Q}^{-1} B\right)$. For $n \in \mathbb{N}$ the measure $\mu$ is conditionally invariant for $S_{Q}^{n}$ with constant $\rho^{n}$ and, in particular, $\rho^{-n} \mu$ is a probability measure on $S_{Q}^{-n}(\mathcal{U} \times Q)$. We construct certain partitions for subsets of $\mathcal{U} \times Q$ whose entropy with respect to $\mu$ will be used to define the metric invariance entropy.

Recall the following definition from reference [6], cf. also [3].
Definition 1: For a subset $Q \subset M$ an invariant $Q$-partition has the form $\mathcal{C}_{\tau}(\mathcal{P}, F)$ where $\tau \in \mathbb{N}, \mathcal{P}$ is a finite partition of $Q$ into Borel measurable sets and $F: \mathcal{P} \rightarrow \Omega^{\tau}$ is a map assigning to each set $P$ in $\mathcal{P}$ a control function such that $\varphi(k, P, F(P)) \subset Q$ for all $k \in\{1, \ldots, \tau\}$.

In the following, we fix an invariant $Q$-partition $\mathcal{C}_{\tau}=$ $\mathcal{C}_{\tau}(\mathcal{P}, F)$ with $\mathcal{P}=\left\{P_{1}, \ldots, P_{q}\right\}$. Abbreviate $F_{i}:=$ $F\left(P_{i}\right) \in \Omega^{\tau}, i=1, \ldots, q$, and define for every word $a:=\left[a_{0}, a_{1}, \ldots, a_{n-1}\right], n \in \mathbb{N}$, with $a_{j} \in\{1, \ldots, q\}$ a control function $u_{a}$ on $\{0, \ldots, n \tau-1\}$ by applying these feedback maps one after the other: for $i=0, \ldots, n-1$ and $k=0, \ldots, \tau-1$

$$
\left(u_{a}\right)_{i \tau+k}:=\left(F_{a_{i}}\right)_{k} .
$$

We also write $u_{a}:=\left(F_{a_{0}}, F_{a_{1}}, \ldots, F_{a_{n-1}}\right)$. A word $a$ is called admissible for $\mathcal{C}_{\tau}$ if there exists a point $x \in Q$ with

$$
\varphi\left(j \tau, x, u_{a}\right) \in P_{a_{j}} \text { for } j=0,1, \ldots, n-1 .
$$

Note that $\varphi\left(k, x, u_{a}\right) \in Q$ for $k=0, \ldots, n \tau$. If $\mathcal{C}_{\tau}$ is clear from the context, we just say that $a$ is admissible. The admissible words describe the possible sequences of partition elements (or "itineraries") under the feedbacks associated with $\mathcal{C}_{\tau}$. For $P \in \mathcal{P}$ we define

$$
A(P)=\{(u, x) \in \mathcal{U} \times P \mid \varphi(k, P, u) \subset Q, k=1, \ldots, \tau\}
$$

and

$$
\mathfrak{A}=\mathfrak{A}\left(\mathcal{C}_{\tau}\right)=\{A(P) \mid P \in \mathcal{P}\}, \mathcal{A}=\bigcup_{P \in \mathcal{P}} A(P) .
$$

This union is disjoint, hence $\mathfrak{A}$ is a partition of $\mathcal{A}$, which, in general, is a proper subset of $\mathcal{U} \times Q$.

A sequence $\left(A_{0}, \ldots, A_{n-1}\right)$ of sets in $\mathfrak{A}$ is called admissible, if there is an admissible word $a=\left[a_{0}, \ldots, a_{n-1}\right]$ of length $n$ with $A_{i}:=A\left(P_{a_{i}}\right)$ for all $i$. Then the set

$$
B_{a}=A_{0} \cap \ldots \cap S_{Q}^{-(n-1) \tau} A_{n-1} \in \bigvee_{i=0}^{n-1} S_{Q}^{-i \tau} \mathfrak{A}
$$

is nonvoid and the collection of all these sets is

$$
\mathfrak{A}_{n}:=\left\{B_{a} \in \bigvee_{i=0}^{n-1} S_{Q}^{-i \tau} \mathfrak{A} \mid a \text { admissible }\right\}
$$

and their union is

$$
\mathcal{A}_{n}:=\bigcup_{B_{a} \in \mathfrak{A}_{n}} B_{a} \subset S_{Q}^{-(n-1) \tau}(\mathcal{U} \times Q) .
$$

Observe that $\mathfrak{A}_{1}=\mathfrak{A}$ and that $\mathfrak{A}_{n}$ is a partition of $\mathcal{A}_{n}$.

By [3, Lemma 3.3], one has for all $n, m \in \mathbb{N}$

$$
\mathfrak{A}_{n+m} \subset \mathfrak{A}_{n} \vee S_{Q}^{-n \tau} \mathfrak{A}_{m}
$$

The converse inclusion does not hold, since not every admissible word of length $m$ may occur as final piece of an admissible word of length $n+m$. Note that every word of length $n$ can be extended to a word of length $n+1$. In fact, for $x \in Q$ with (6) one obtains $\varphi\left(n \tau, x, u_{a}\right) \in Q$, hence there is $P \in \mathcal{P}$ with $\varphi\left(n \tau, x, u_{a}\right) \in P=: P_{a_{n}}$ and the word $\left[a, a_{n}\right]$ is admissible. Observe also that this extension will, 
in general, not be unique, since it depends on the choice of $x$.

Consider the entropy $H_{\rho^{-(n-1) \tau} \mu}\left(\mathfrak{A}_{n}\right)$ of $\mathfrak{A}_{n}$ with respect to the probability measure $\rho^{-(n-1) \tau} \mu$ on $S_{Q}^{-(n-1) \tau}(\mathcal{U} \times Q)$ and then take the average of the required information as time tends to $\infty$ to get the entropy of $\mathcal{C}_{\tau}$,

$$
h_{\mu}\left(\mathcal{C}_{\tau}, Q\right):=\liminf _{n \rightarrow \infty} \frac{1}{n \tau} H_{\rho^{-(n-1) \tau} \mu}\left(\mathfrak{A}_{n}\left(\mathcal{C}_{\tau}\right)\right) .
$$

Definition 2: The $\mu$-invariance entropy for control system (1) described by the map $S$ (cf. (3)) is defined by

$$
h_{\mu}(Q, S)=\liminf _{\tau \rightarrow \infty} \inf _{\mathcal{C}_{\tau}} h_{\mu}\left(\mathcal{C}_{\tau}, Q\right),
$$

where the infimum is taken over all invariant $Q$-partitions $\mathcal{C}_{\tau}(\mathcal{P}, F)$ with fixed $\tau \in \mathbb{N}$.

Definition (10) guarantees that $\tau \rightarrow \infty$ which will be needed below. For topological invariance entropy, one takes instead an infimum over all invariant partitions $\mathcal{C}_{\tau}=(\mathcal{P}, F)$ with $\tau \in \mathbb{N}$, partitions $\mathcal{P}$ and maps $F: \mathcal{P} \rightarrow \Omega^{\tau}$. Then it is a consequence that it suffices to take the limit for $\tau \rightarrow \infty$; cf. Theorem 4, below.

We note the following proposition which follows by a variant of a standard lemma in this context.

Proposition 3: The entropy with respect to $\mu$ satisfies

$$
H_{\mu}\left(\mathfrak{A}_{n+m}\right) \leq H_{\mu}\left(\mathfrak{A}_{n}\right)+\rho^{n \tau} H_{\mu}\left(\mathfrak{A}_{m}\right) \text { for } n, m \in \mathbb{N},
$$

and hence $\frac{1}{n} H_{\mu}\left(\mathfrak{A}_{n}\right) \rightarrow 0$ for $n \rightarrow \infty$.

This proposition shows that metric invariance entropy can not reasonably be defined using just the conditionally invariant measure $\mu$. Instead the renormalized measures $\rho^{-n \tau} \mu$ are needed.

In [6, Definition 2.2 and Proposition 2.3(ii)] the following notion is considered. For system (1) let $Q \subset M$ be a compact controlled invariant set, i.e., for every $x \in Q$ there is $\omega_{x} \in \Omega$ with $f\left(x, \omega_{x}\right) \in Q$. For $\tau \in \mathbb{N}$ a set $\mathcal{R} \subset \mathcal{U}$ is called $(\tau, Q)$-spanning if for all $x \in Q$ there is $u \in \mathcal{R}$ with $\varphi(n, x, u) \in Q$ for all $n=1, \ldots, \tau$. Denote by $r_{i n v}(\tau, Q)$ the minimal number of elements such a set can have (if no finite $(\tau, Q)$-spanning set exists, $\left.r_{i n v}(\tau, Q):=\infty\right)$. The topological invariance entropy is defined by

$$
h_{i n v}(Q, S):=\lim _{\tau \rightarrow \infty} \frac{1}{\tau} \log r_{i n v}(\tau, Q) .
$$

For an invariant partition $\mathcal{C}_{\tau}$ write $\# \mathcal{W}_{N}\left(\mathcal{C}_{\tau}\right)$ for the number of elements in the set $\mathcal{W}_{N}\left(\mathcal{C}_{\tau}\right)$ of all admissible words of length $N$. Define the entropy of $\mathcal{C}_{\tau}$ by

$$
h\left(\mathcal{C}_{\tau}\right):=\lim _{N \rightarrow \infty} \frac{\log \# \mathcal{W}_{N}\left(\mathcal{C}_{\tau}\right)}{N \tau}=\inf _{N \in \mathbb{N}} \frac{\log \# \mathcal{W}_{N}\left(\mathcal{C}_{\tau}\right)}{N \tau} .
$$

The following characterization of topological invariance entropy is given in [6, Theorem 2.3 and its proof].

Theorem 4: For a compact and controlled invariant set $Q$ it holds that

$$
h_{\text {inv }}(Q, S)=\inf _{\mathcal{C}_{\tau}} h\left(\mathcal{C}_{\tau}\right)=\liminf _{\tau \rightarrow \infty} \inf _{\mathcal{C}_{\tau}} h\left(\mathcal{C}_{\tau}\right),
$$

where the infimum is taken over all invariant $Q$-partitions $\mathcal{C}_{\tau}=\mathcal{C}_{\tau}(\mathcal{P}, F)$.
The following result relates metric and topological invariance entropy.

Proposition 5: Let $Q$ be a compact and controlled invariant set $Q$. Then for every conditionally invariant measure $\mu$ on $Q$

$$
h_{\mu}(Q, S) \leq h_{\text {inv }}(Q, S) .
$$

Proof: Let $\mathcal{C}_{\tau}(\mathcal{P}, F)$ be an invariant partition. Then $\# \mathfrak{A}_{n}\left(\mathcal{C}_{\tau}\right)=\# \mathcal{W}_{N}\left(\mathcal{C}_{\tau}\right)$ and for every $n \in \mathbb{N}$ a standard property of entropy yields

$$
H_{\rho^{-(n-1) \tau} \mu}\left(\mathfrak{A}_{n}\left(\mathcal{C}_{\tau}\right)\right) \leq \log \# \mathfrak{A}_{n}\left(\mathcal{C}_{\tau}\right) .
$$

It readily follows that $h_{\mu}(Q, S) \leq h_{\text {inv }}(Q, S)$, since

$$
\begin{aligned}
h_{\mu}\left(\mathcal{C}_{\tau}, Q\right) & =\liminf _{n \rightarrow \infty} \frac{1}{n \tau} H_{\rho^{-(n-1) \tau} \mu}\left(\mathfrak{A}_{n}\left(\mathcal{C}_{\tau}\right)\right) \\
& \leq \lim _{N \rightarrow \infty} \frac{\log \# \mathcal{W}_{N}\left(\mathcal{C}_{\tau}\right)}{N \tau}=h\left(\mathcal{C}_{\tau}\right) .
\end{aligned}
$$

\section{INVARIANCE ENTROPY AND RELATIVE INVARIANCE}

In this section, we show that the metric invariance entropy of $Q$ is already determined on certain subsets $K$ of $Q$.

We call a subset $K \subset Q$ invariant in $Q$, if $x \in K$ and $\varphi(k, x, u) \notin K$ for some $k \in \mathbb{N}$ and $u \in \mathcal{U}$ implies $\varphi(k, x, u) \notin Q$.

First we determine a relation between invariant partitions of $K$ and $Q$.

Lemma 6: Let $K$ be a closed invariant subset in the closed set $Q$.

(i) Every invariant $Q$-partition $\mathcal{C}_{\tau}\left(\mathcal{P}^{Q}, F^{Q}\right)$ induces an invariant $K$-partition $\mathcal{C}_{\tau}\left(\mathcal{P}^{K}, F^{K}\right)$ given by

$$
\mathcal{P}^{K}=\left\{P \cap K \mid P \in \mathcal{P}^{Q}\right\} \text { and } F^{K}(P \cap K)=F^{Q}(P) .
$$

(ii) Assume that there are a finite measurable cover of $Q$ by sets $V^{1}, \ldots, V^{N}$, control functions $u^{1}, \ldots, u^{N} \in \mathcal{U}$ and times $\tau^{1}, \ldots, \tau^{N} \in \mathbb{N}$ such that for all $x \in V^{j}$, all $j=1, \ldots, N$ and all $k=0, \ldots, \tau^{j}$

$$
\varphi\left(k, x, u^{j}\right) \in Q \text { and } \varphi\left(\tau^{j}, x, u^{j}\right) \in K .
$$

Then every invariant $K$-partition $\mathcal{C}_{\tau}\left(\mathcal{P}^{K}, F^{K}\right)$ with $\tau \geq \bar{\tau}:=\max _{j=1, \ldots, N} \tau^{j}$ can be extended to an invariant $Q$-partition $\mathcal{C}_{\tau}\left(\mathcal{P}^{Q}, F^{Q}\right)$ such that $\mathcal{P}^{K} \subset \mathcal{P}^{Q}$ and $F^{Q}(P)=F^{K}(P)$ for $P \in \mathcal{P}^{K}$ and $\varphi\left(\tau, P, F^{Q}(P)\right) \subset$ $K$ for all $P \in \mathcal{P}^{Q}$.

Proof:

(i) Since $K$ is invariant in $Q$ it follows for $P \in \mathcal{P}^{Q}$ from $P \cap K \subset K$ and $\varphi\left(k, P \cap K, F^{Q}(P)\right) \subset Q$ that $\varphi\left(k, P \cap K, F^{Q}(P)\right) \subset K$. Thus $\mathcal{C}_{\tau}\left(\mathcal{P}^{K}, F^{K}\right)$ is an invariant $K$-partition.

(ii) Let $\mathcal{C}_{\tau}\left(\mathcal{P}^{K}, F^{K}\right)$ be an invariant $K$-partition with $\tau \geq$ $\bar{\tau}$. The cover of $Q$ induces a finite partition $\mathcal{P}_{1}$ of $Q \backslash \bar{K}$ such that for every $P_{1}^{j} \in \mathcal{P}_{1}$ the control $F_{1}\left(P_{1}^{j}\right):=$ $\left(u_{0}^{j}, \ldots, u_{\tau^{j}-1}^{j}\right) \in \Omega^{\tau}$ satisfies $\varphi\left(k, P_{1}^{j}, F_{1}\left(P_{1}^{j}\right)\right) \subset Q$ for all $k=0, \ldots, \tau^{j}$. In fact, we may define $P_{1}^{1}:=$ $(Q \backslash K) \cap V_{1}$ and

$$
P_{1}^{j}:=\left[(Q \backslash K) \cap V^{j}\right] \backslash \bigcup_{i<j} P_{1}^{i} \text { for } j>1 .
$$


Then $\varphi\left(k, P_{1}^{j}, F_{1}\left(P_{1}^{j}\right)\right) \subset Q$ for all $k=0, \ldots, \tau^{j}$ and $\varphi\left(\tau^{j}, P_{1}^{j}, F_{1}\left(P_{1}^{j}\right)\right) \subset K$ since $P_{1}^{j} \subset V^{j}$. In particular, there is $P^{i} \in \mathcal{P}^{K}$ with $\varphi\left(\tau^{j}, P_{1}^{j}, F_{1}\left(P_{1}^{j}\right)\right) \in P^{i}$ and hence for all $x \in P_{1}^{j}$ and all $k=0, \ldots, \tau$

$$
\varphi\left(k, \varphi\left(\tau^{j}, x, F_{1}\left(P_{1}^{j}\right)\right), F^{K}\left(P^{i}\right)\right) \in K .
$$

One obtains an invariant $Q$-partition $\mathcal{C}_{\tau}\left(\mathcal{P}^{Q}, F^{Q}\right)$ in the following way: The partition consists of the sets in $\mathcal{P}^{K}$ together with all (nonvoid) sets of the form

$$
P^{i, j}:=\left\{x \in P_{1}^{j} \mid \varphi\left(\tau^{j}, x, F_{1}\left(P_{1}^{j}\right)\right) \in P^{i}\right\}
$$

with feedbacks defined as follows: For $P^{i} \in \mathcal{P}^{K}$ let $F^{Q}\left(P^{i}\right)=F^{K}\left(P^{i}\right)$. Furthermore, denoting the controls associated with the feedbacks $F^{K}\left(P^{i}\right), P^{i} \in$ $\mathcal{P}^{K}$, by $v_{k}^{i} \in \Omega, k=0, \ldots, \tau-1$, define

$$
F^{Q}\left(P^{i, j}\right)_{k}=\quad \begin{array}{ccc}
u_{k}^{j} & \text { for } & k=0, \ldots, \tau^{j}-1 \\
v_{k-\tau^{j}}^{i} & \text { for } & k=\tau^{j}, \ldots, \tau-1 .
\end{array}
$$

This is well defined, since $\tau-\tau^{j} \geq 0$ and hence $\mathcal{C}_{\tau}\left(\mathcal{P}^{Q}, F^{Q}\right)$ is an invariant $Q$-partition.

The next theorem shows when the invariance $\mu$-entropy is already determined on a subset $K$ of $Q$.

Theorem 7: Consider control system (1) described by the map $S$. Let $K \subset Q$ be invariant in $Q$ and fix a conditionally invariant measure $\mu$ for $Q$.

(i) Then the $\mu$-invariance entropy of $K$ is bounded above by the $\mu$-invariance entropy of $Q, h_{\mu}(K, S) \leq h_{\mu}(Q, S)$.

(ii) If the assumption in Lemma 6(ii) is satisfied, then $h_{\mu}(Q, S)=h_{\mu}(K, S)$.

Proof: A proof of assertion (ii) will be given elsewhere. For the proof of (i) let $\mathcal{C}_{\tau}^{Q}=\mathcal{C}_{\tau}\left(\mathcal{P}^{Q}, F^{Q}\right)$ be an invariant $Q$-partition and consider the associated invariant $K$-partition $\mathcal{C}_{\tau}^{K}=\mathcal{C}_{\tau}\left(\mathcal{P}^{K}, F^{K}\right)$ according to Lemma 6. We will show that $h_{\mu}\left(\mathcal{C}_{\tau}^{K}, K\right) \leq h_{\mu}\left(\mathcal{C}_{\tau}^{Q}, Q\right)$. Then, taking first the infimum over all invariant $Q$-partitions $\mathcal{C}_{\tau}^{Q}$ and then over all invariant $K$-partitions $\mathcal{C}_{\tau}^{K}$ and, finally, the limit inferior for $\tau \rightarrow$ $\infty$, one concludes that $h_{\mu}(K, S) \leq h_{\mu}(Q, S)$ holds, i.e., assertion (i).

For $P \in \mathcal{P}^{Q}$ consider $(u, x) \in A\left(P, \mathcal{C}_{\tau}^{Q}\right)$, hence $\varphi(k, P, u) \subset Q$ for all $k=0, \ldots, \tau$. If $x \in P \cap K$, then conditional invariance of $K$ in $Q$ implies that $\varphi(k, x, u) \subset K$ for all $k=1, \ldots, \tau$. It follows for all $P \cap K \in \mathcal{P}^{K}$

$$
A\left(P \cap K, \mathcal{C}_{\tau}^{K}\right)=A\left(P, \mathcal{C}_{\tau}^{Q}\right) \cap(\mathcal{U} \times K)
$$

showing that $\mathfrak{A}^{K}=\mathfrak{A}^{Q} \cap(\mathcal{U} \times K)$.

If $\left(P_{0} \cap K, \ldots, P_{n-1} \cap K\right)$ is a $\mathcal{C}_{\tau}^{K}$-admissible partition sequence, then $\left(P_{0}, \ldots, P_{n-1}\right)$ is a $\mathcal{C}_{\tau}^{Q}$-admissible partition sequence and hence a $\mathcal{C}_{\tau}^{K}$-admissible word $a$ is also $\mathcal{C}_{\tau}^{Q}$ admissible. The corresponding $\mathcal{C}_{\tau}^{Q}$-admissible set

$$
B_{a}=A_{a_{0}} \cap S_{Q}^{-\tau} A_{a_{1}} \cap \ldots \cap S_{Q}^{-(n-1) \tau} A_{a_{n-1}} \in \mathfrak{A}_{n}^{Q}
$$

yields the $\mathcal{C}_{\tau}^{K}$-admissible set in $\mathfrak{A}_{n}^{K}$

$$
\begin{aligned}
& B_{a} \cap(\mathcal{U} \times K) \\
& =\left[A_{a_{0}} \cap(\mathcal{U} \times K)\right] \cap S_{Q}^{-\tau}\left[A_{a_{1}} \cap(\mathcal{U} \times K)\right] \cap \ldots \\
& \left.\quad \ldots \cap S_{Q}^{-(n-1) \tau} A_{a_{n-1}} \cap(\mathcal{U} \times K)\right] .
\end{aligned}
$$

In fact, invariance of $K$ in $Q$ implies for all $(u, x) \in B_{a} \cap$ $(\mathcal{U} \times K)$ that $\varphi(k, x, u) \in K$ for all $k=0, \ldots,(n-1) \tau$ and, in particular, $\varphi(i \tau, x, u) \in P_{a_{i}} \cap K$ for $i=0, \ldots, n-1$.

It follows that

$$
\mathfrak{A}_{n}^{K} \subset \mathfrak{A}_{n}^{Q} \cap(\mathcal{U} \times K) \text { for all } n .
$$

This inclusion may be proper for $n>1$ : For a $\mathcal{C}_{\tau}^{Q}$-admissible word $a=\left[a_{0}, \ldots, a_{n-1}\right]$ of length $n$ with $B_{a} \cap(\mathcal{U} \times K) \neq \emptyset$ there may not exist $x \in K$ with $\left(u_{a}, x\right) \in B_{a}$. Thus an intersection $B_{a} \cap(\mathcal{U} \times K), B \in \mathfrak{A}_{n}^{Q}$, may be nonvoid, but not $\mathcal{C}_{\tau}^{K}$-admissible.

First consider $B \in \mathfrak{A}_{n}^{Q}$ with $\rho^{-(n-1) \tau} \mu(B) \leq e^{-1}$. Then also $\rho^{-(n-1) \tau} \mu(B \cap(\mathcal{U} \times K)) \leq e^{-1}$ and it follows that

$$
\left.\phi \quad \rho^{-(n-1) \tau} \mu(B)\right) \leq \phi\left(\rho^{-(n-1) \tau} \mu(B \cap(\mathcal{U} \times K))\right),
$$

since $\phi$ is monotonically decreasing on $\left[0, e^{-1}\right]$. For every $n \in \mathbb{N}$ there are at most three sets $B \in \mathfrak{A}_{n}^{Q}$ with $\rho^{-(n-1) \tau} \mu(B) \geq e^{-1}$, since they are disjoint and the sum of the measures of four sets $B \subset \mathcal{A}_{n}^{Q} \subset S_{Q}^{-(n-1) \tau}(\mathcal{U} \times Q)$ with this property would be greater than or equal to $4 e^{-1}>$ $1=\rho^{-(n-1) \tau} \mu\left(S_{Q}^{-(n-1) \tau}(\mathcal{U} \times Q)\right)$. Let

$$
\mathfrak{A}_{n}^{Q, b i g}:=\left\{B \in \mathfrak{A}_{n}^{Q} \mid \rho^{-(n-1) \tau} \mu(B) \geq e^{-1}\right\} .
$$

Then $\# \mathfrak{A}_{n}^{Q, b i g} \leq 3$ and, using $\phi(x) \geq \phi\left(e^{-1}\right)=-e^{-1}, x \in$ $[0,1]$, it follows that

$$
\left.\sum_{B \in \mathfrak{A}_{n}^{Q, b i g}} \phi \rho^{-(n-1) \tau} \mu(B \cap(\mathcal{U} \times K))\right) \geq-3 / e .
$$

We find, using $\phi(x) \leq 0$ and (11),

$$
\begin{aligned}
& -H_{\rho^{-(n-1) \tau} \mu}\left(\mathfrak{A}_{n}^{Q}\right) \\
& =\sum_{B \in \mathfrak{A}_{n}^{Q}} \phi \rho^{-(n-1) \tau} \mu(B) \\
& \left.\leq \sum_{B \in \mathfrak{A}_{n}^{Q} \backslash \mathfrak{A}_{n}^{Q, b i g}} \phi \rho^{-(n-1) \tau} \mu(B \cap(\mathcal{U} \times K))\right) \\
& \left.=\sum_{B \in \mathfrak{A}_{n}^{Q}} \phi \rho^{-(n-1) \tau} \mu(B \cap(\mathcal{U} \times K))\right) \\
& \left.\quad-\sum_{B \in \mathfrak{A}_{n}^{Q, b i g}} \phi \rho^{-(n-1) \tau} \mu(B \cap(\mathcal{U} \times K))\right) \\
& \left.\leq \sum_{B \in \mathfrak{A}_{n}^{Q}} \rho^{-(n-1) \tau} \mu(B \cap(\mathcal{U} \times K))\right)+3 / e \\
& \left.=-H_{\rho^{-(n-1) \tau} \mu}\left(\mathfrak{A}_{n}^{K}\right)\right)+3 / e .
\end{aligned}
$$

It follows that

$$
\begin{aligned}
\left.h_{\mu}\left(\mathfrak{A}^{Q}\right)\right) & =\liminf _{n \rightarrow \infty} \frac{1}{n \tau} H_{\rho^{-(n-1) \tau} \mu}\left(\mathfrak{A}_{n}^{Q}\right) \\
& \left.\geq \liminf _{n \rightarrow \infty} \frac{1}{n \tau} H_{\rho^{-(n-1) \tau} \mu}\left(\mathfrak{A}_{n}^{K}\right)-3 / e\right] \\
& =h_{\mu}\left(\mathfrak{A}^{K}\right)
\end{aligned}
$$

concluding the proof of Theorem 7(i). 


\section{INVARIANT $W$-CONTROL SETS}

Next we construct relatively invariant subsets of $Q$ with the properties in Theorem 7 using controllability properties. Again we consider system (1) and introduce some notation for controllability properties within the open set $W:=\operatorname{int} Q$.

Write $\varphi_{W}(n, x, u)=\varphi(n, x, u)$ if $\varphi(k, x, u) \in W$ for $k=0, \ldots, n \in \mathbb{N}$. Define the reachable and controllable set within $W$ by

$$
\begin{aligned}
& \mathcal{O}_{W}^{+}(x)=\left\{\varphi_{W}(n, x, u) \text { with } n \in \mathbb{N} \text { and } u \in \mathcal{U}\right\}, \\
& \mathcal{O}_{W}^{-}(x)=\left\{y \in W \mid \exists n \in \mathbb{N} \exists u \in \mathcal{U}: \varphi_{W}(n, y, u)=x\right\} .
\end{aligned}
$$

We assume that the system is accessible, i.e.,

$$
\operatorname{int} \mathcal{O}_{W}^{ \pm}(x) \neq \emptyset \text { for all } x \in W \text {. }
$$

Definition 8: A subset $D \subset W$ is called a $W$-control set if (i) $D \subset \operatorname{cl}_{W} \mathcal{R}^{W}(x)$ for all $x \in D$, (ii) there is $x \in$ $D$ with $x \in \operatorname{int} \mathcal{O}_{W}^{-}(x)$ and (iii) $D$ is maximal with these properties. A $W$-control set $D$ is called invariant, if $\operatorname{cl}_{W} D=$ $\mathrm{cl}_{W} \mathcal{R}^{W}(x)$ for all $x \in D$.

Here the closures are taken with respect to $W$.

The following theorem constructs a set $K$ which is invariant in $Q$ using invariant $W$-control sets. It follows using results in [14].

Theorem 9: Assume that the state space $M$ is a connected analytic Riemannian manifold and $W \subset M$ is an open, connected and relatively compact subset. Let the control range $\Omega \subset \mathrm{cl} \operatorname{int} \Omega \subset \mathbb{R}^{m}$ and $f: M \times \hat{\Omega} \rightarrow M$ be real analytic, where $\hat{\Omega}$ is an open set containing $\Omega$. Furthermore, suppose that

$$
\Omega_{s u b}:=\{\omega \in \Omega \mid f(\cdot, \omega) \text { is submersive }\}
$$

is the complement of a proper analytic subset in $\hat{\Omega} \cap \Omega$.

(i) Then the following statements are equivalent:

(a) There are at least one and at most finitely many invariant $W$-control sets $D_{1}, \ldots, D_{\ell}, 1 \leq \ell<\infty$, and for every $x \in W$ there is $D_{i}$ with

$$
\mathcal{O}_{W}^{+}(x) \cap D_{i} \neq \emptyset \text {. }
$$

(b) There is a compact set $F \subset W$ with

$$
F \cap \operatorname{clO}_{W}^{+}(x) \neq \emptyset \text { for all } x \in W .
$$

(ii) Suppose that the statement in (a) (or (b)) is valid and that $Q$ is equal to the closure of $W=\operatorname{int} Q$. Define

$$
K=\bigcup_{i=1}^{l} \operatorname{cl} D_{i}
$$

and assume that $f(K, \Omega) \cap(\partial Q \backslash K)=\emptyset$.

Then $K$ is invariant in $Q$, and for every conditionally invariant measure $\mu$ on $Q$

$$
h_{\mu}(Q, S)=h_{\mu}(K, S) \geq \sum_{i=1}^{l} h_{\mu}\left(\operatorname{cl} D_{i}, S\right) \text {. }
$$

Remark 10: In the continuous-time case a similar result for the topological invariance entropy has been shown in [4].

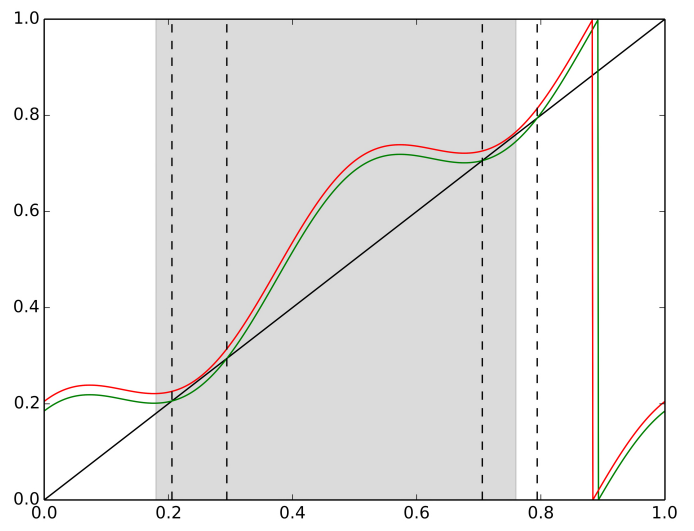

Fig. 1. Graphs for $f_{\alpha}(x, \pm 1), x \in[0,1]$ with $\sigma=0.1, A=0.01$, $\alpha=0.095$

\section{AN EXAMPLE}

Consider the family of control systems depending on a real parameter $\alpha$ given by $f_{\alpha}: \mathbb{R} / \mathbb{Z} \times[-1,1] \rightarrow \mathbb{R} / \mathbb{Z}$,

$$
f_{\alpha}(x, \omega)=x+\sigma \cos (4 \pi x)+A \omega+\alpha \bmod 1 .
$$

Suppose that the amplitudes $A$ and $\sigma$ take on small positive values. For $\alpha_{0}=\sigma-A$ the extremal graph $f_{\alpha_{0}}(\cdot, 1)$ is tangent to the diagonal at two points with abscissa 0.25 and 0.75 , respectively.

For $\alpha>\alpha_{0}$ the only control set is $\mathbb{R} / \mathbb{Z}$. The situation for the parameters $\sigma=0.1, A=0.01$ and $\alpha=0.095$ is illustrated in Fig. 1. Here $\alpha$ is greater than the critical value $\alpha_{0}=0.090$. For $W=(0.18,0.76)$ there are two $W$-control sets $D_{1}^{\alpha}$ and $D_{2}^{\alpha}$, where only the one to the right (say $D_{2}^{\alpha}$ ) is invariant in $W$. In Fig. 1 the dashed lines indicate the boundaries of the two $W$-control sets and the shaded region corresponds to the set $W$.

Let a probability measure $\nu$ on $\Omega=[-1,1]$ be given with a density with respect to Lebesgue measure and suppose that there is a $\gamma>0$ such that $p(x, Q) \geq \gamma>0$ for all $x \in Q=$ $[0.18,0.76]$. Then [3, Theorem 2.9] implies the existence of a quasi-stationary measure with $0<\rho<1$. If we take the uniform distribution on $\Omega$ these condition are satisfied. Hence one finds a conditionally invariant measure $\mu^{\alpha}=\nu^{\mathbb{N}} \times \eta^{\alpha}$ on $\mathcal{U} \times Q$. Theorem 7 shows that for every quasi-stationary measure $\eta^{\alpha}$ of $Q=\operatorname{cl} W$ the invariance entropy for $\mu^{\alpha}$ on $Q$ coincides with the invariance entropy of the closure of the invariant $W$-control set $D_{2}^{\alpha}$.

On the other hand, numerical experiments seem to indicate that the support of this quasi-stationary measure is the union of $\operatorname{cl} D_{1}^{\alpha}$ and $\operatorname{cl} D_{2}^{\alpha}$.

\section{CONCLUSIONS}

The results above show that the metric invariance entropy of a subset $Q$ of the state space is already determined on a subset $K$ that can be characterized using controllability properties. Essentially, one needs that all points in $Q$ can be 
steered into $K$ with a finite number of control functions and that the system can leave $K$ only if it also leaves $Q$.

\section{REFERENCES}

[1] F. Albertini and E. D. Sontag. Discrete-time transitivity and accessibility: Analytic systems. SIAM J. Control Optim., 31:1599-1622, 1993.

[2] P. Collett, S. Martinez, and J. San Martin. Quasi-Stationary Distributions: Markov Chains, Diffusions, and Dynamical Systems. SpringerVerlag, Berlin, 2013.

[3] F. Colonius. Metric invariance entropy and conditionally invariant measures. Ergodic Theory and Dynamical Systems, 2016. to appear.

[4] F. Colonius and R. Lettau. Relative controllability properties. IMA Journal of Mathematical Control and Information, 2015. doi:10.1093/imamci/dnv004.

[5] D.F. Delchamps. Stabilizing a linear system with quantized state feedback. IEEE Trans. Aut. Control, 35:916-924, 1990.

[6] C. Kawan. Invariance Entropy for Deterministic Control Systems. An Introduction. Springer-Verlag, 2013.

[7] P.G. Mehta, U. Vaidya, and A. Banaszuk. Markov chains, entropy, and fundamental limitations in nonlinear stabilization. IEEE Trans. Aut. Control, 53(3):784-791, 2008.

[8] S. Méléard and D. Villemonais. Quasi-stationary distributions and population processes. Probability Surveys, 9:340-410, 2012.

[9] G.N. Nair, R. J. Evans, I.M.Y. Mareels, and W. Moran. Topological feedback entropy and nonlinear stabilization. IEEE Trans. Aut. Control, 49:1585-1597, 2004.

[10] M. Patrão and L.A.B. San Martin. Semiflows on topological spaces: Chain transitivity and semigroups. J. Dyn. Diff. Equations, 19:155180, 2007.

[11] E. Sontag and F. Wirth. Remarks on universal nonsingular controls for discrete-time systems. Sys. Control Lett., 33:81-88, 1998.

[12] M. Viana and K. Oliveira. Foundations of Ergodic Theory. Cambridge University Press, 2016.

[13] P. Walters. An Introduction to Ergodic Theory. Springer-Verlag, 1982.

[14] F. Wirth. Dynamics and controllability of nonlinear discrete-time control systems. In Preprints of the 4th IFAC Nonlinear Control Systems Design Symposium, NOLCOS98, Enschede, The Netherlands, pages 269-275, 1998. 